\title{
Manufacturing Of Aluminum Coating On 3D-Printed Onyx With Cold Spray Technology
}

Roberta Della Gatta, Antonello Astarita, Domenico Borrelli, Antonio Caraviello, Francesco Delloro, Patrizio Lomonaco, Ilaria Papa, Alessia Serena Perna, Raffaele Sansone and Antonio Viscusi

Roberta Della Gatta. Department of Chemical, Materials and Production Engineering, University of Naples Federico II, Piazzale Tecchio 80, Naples, Italy.

Corresponding author: roberta.dellagatta@unina.it

Antonello Astarita. Department of Chemical, Materials and Production Engineering, University of Naples Federico II, Piazzale Tecchio 80, Naples, Italy.

Domenico Borrelli. Sophia High tech, Via Romani 228, 80048, Sant'Anastasia NA, Italy.

Antonio Caraviello. Sophia High tech, Via Romani 228, 80048, Sant'Anastasia NA, Italy.

Francesco Delloro. Département Mécanique et Matériaux (MINES ParisTech), Paris, France.

Patrizio Lomonaco. Département Mécanique et Matériaux (MINES ParisTech), Paris, France

Ilaria Papa. Department of Chemical, Materials and Production Engineering, University of Naples Federico II, Piazzale Tecchio 80, Naples, Italy.

Alessia Serena Perna. Department of Chemical, Materials and Production Engineering, University of Naples Federico II, Piazzale Tecchio 80, Naples, Italy.

University of Bergamo, Bergamo, Italy.

Raffaele Sansone. Sophia High tech, Via Romani 228, 80048, Sant'Anastasia NA, Italy.

Antonio Viscusi. Department of Chemical, Materials and Production Engineering, University of Naples Federico II, Piazzale

Tecchio 80, Naples, Italy

Abstract. Composite materials are widely used as main parts and structural components in different fields, especially for automotive and military applications. Although these materials supply different advantages comparing to the metals, their implementation in engineering applications is limited due to low electrical and thermal properties and low resistance to erosion. To enhance these above-mentioned properties, the metallization of composite materials by creating a thin metal film on their surface can be achieved. Among different coating deposition techniques, Cold Spray appears to be the most suitable one for the metallization of temperature-sensitive materials such as polymers and composites with a thermoplastic matrix. This process relies on kinetic energy for the formation of the coating rather than on thermal energy and consequent erosion and degradation of the polymer-based composite can be avoided. In the last years, a new method to produce composite materials, as known as Fused Filament Fabrication (FFF), has been developed for industrial applications. This technique consists of a 3D printing process that involves the thermal extrusion of thermoplastic polymer and fibers in the form of filaments from a heated mobile nozzle. The implementation of this new technique is leading to the manufacturing of customized composite materials for the cold spray application. In the presented experimental campaign, Onyx material is used as a substrate. This material is made of Nylon, a thermoplastic matrix, and chopped carbon fibers randomly dispersed in it. Aluminum powders were cold sprayed on the Onyx substrate with a low-pressure cold spray (LPCS) system. This study aims to investigate the possibility of the metalizing 3D-printed composite material by cold spray technology. For this purpose, optical and microscopical analyses are carried out. Based on the results, the feasibility of the process and the influence of the morphology of the substrate are discussed, and optimal spraying conditions are proposed.

Keywords. Cold Spray, 3D-printing, Aluminum, Onyx 


\section{Introduction}

In the last years, the metallization of polymer materials has received increasing attention in a wide range of applications, such as in microelectronics or aeronautic industries [1]. To enhance the electrical and thermal properties and the resistance to erosion of these materials, the metallization of their surfaces by creating a thin metal film on their surface can be achieved. For this purpose, several coating technologies have been investigated, such as physical vapor deposition (PVD)[2], chemical vapor deposition (CVD)[3], or plasma-enhanced chemical vapor deposition (PECVD) [4]. Although these techniques can accomplish the metallization of the polymers, the thickness of the deposits could not exceed a few micrometers and the costs of the used equipment are expensive. Among all the other coating techniques proposed, the Cold Spray has been demonstrated to be a feasible approach to metalize polymers and polymeric composites [5] and able to avoid the thermal degradation of the polymers caused by the high operating temperatures. The cold spray technology is a thermal spray process in which particles of various materials, mostly metals, are accelerated by a heated and pressurized gas through a convergent-divergent nozzle to the substrate[6]. The particles are heated-up below the melting point of the material and remain in a solid-state for the entire process. The main characteristic of the cold spray technique is that it relies more on kinetic energy than on thermal energy and that makes this technology suitable for the metallization of temperature-sensitive materials like polymers and composite materials.

Although the cold spray deposition on polymers ad composite materials has been widely studied in the last years $[7,8]$, the bonding mechanisms between the particles and the polymer substrate are not well understood and the influence of the main process parameters is still an object of study. Che et al [9] have assumed that the bonding within sprayed particles and polymer substrates is possible only by mechanical interlocking and the well-known adiabatic shear instability phenomena [6] could not take place due to the different chemical natures of both substrates and powder. Ganesan et al [10] have also proven that mechanical interlocking is facilitated when spraying on thermoplastic polymers instead of thermosetting ones. In fact, when the particles impact a thermoplastic substrate, their energy is dissipated in part for the particle deformation and in part for the substrate deformation of the thermoplastic polymer. Due to the nature of the chemical links between the polymeric chains in thermoplastic materials, the local temperature rising at the particle-polymer interface facilitates the polymer softening and the consequent particle embedment.

Anyway, a lot of studies have been conducted on the influence of different process parameters, but less importance has been given to the manufacturing technique of the substrate which, on the contrary, is relevant for the successful deposition. In fact, as proved by Gillet et al.[11], the different behavior of the particle deformation should be expected when spraying on composite instead of polymer. Also, the morphology of the substrate plays a leading role in the cold spray deposition: when the substrate presents cavities the mechanical interlocking within powders and substrate is facilitated [12].

In industrial applications, different technologies are used to manufacture composite objects, especially with a thermoplastic matrix. Anyway, most of these techniques, such as compression molding [13] cannot ensure full control of the polymer's stratification, the fiber content, the thicknesses of both fibers and matrix layers, and the consequent morphology of the substrate. To overcome these issues, the 3D printing of the polymer or composite substrate can offer many advantages in the fabrication of these components, including high precision, cost-effectiveness, and customized geometry [14]. Among all the different techniques, the Fused Filament Fabrication (FFF) technology is the most reliable to guarantee the above-mentioned requirements. FFF technology allows for the deposition of both matrix and reinforcement in the form of the filament by heated nozzles. As for most additive manufacturing technologies, the manufacturing of the final component is made by a layer-by-layer stratification which requires the implementation of two heated nozzles, one for the matrix and the other for the fibers. In this way, it is possible to control fibers volume, polymer, and fiber layer thickness, and in particular the scan strategy. For the presented experimental campaign, a Markforged X7 machine has been used [15]. A new commercial material, Onyx, has been chosen as feedstock material 
for the substrate. This material is made of Nylon, a thermoplastic polymer, and chopped carbon fibers randomly dispersed in it. Aluminum powders were sprayed on the Onyx substrate with a low-pressure cold spray system.

The object of this study is to investigate the feasibility of the metallization of the presented 3D-printed composite materials employing a low-pressure cold spray technology. For this purpose, optical and microscopical analyses are carried out. The amount of powder deposition has been analyzed through surface coverage quantification. Based on the results, the feasibility of the process and the influence of the substrate are discussed, and optimal spraying conditions, including standoff distance and gas inlet temperature, are proposed.

\section{Materials and Methods}

\subsection{Manufacturing of the Composite Laminates}

The substrate used for the presented experimental campaign was manufactured by the means of the 3D printing machine Markforged X7. With this equipment, the manufacturing of polymer and composites objects is made possible with the Fused Filament Fabrication (FFF) technique. The FFF is a 3D printing technology that belongs to the largest additive manufacturing group where both filament and polymer feedstock materials are in a filament form. By the means of heated nozzles, filaments are fused with a layer-by-layer stratification to fabricate a solid part.

For the substrate, an Onyx filament of about $150 \mu \mathrm{m}$ in diameter was used as feedstock materials. Onyx is a composite material made of Nylon, thermoplastic matrix, and chopped carbon fibers. The presence of the carbon fibers gives homogeneity to the panel and guarantees higher stiffness to the substrate [16].

As a substrate, a panel of $80 \times 80 \mathrm{~mm}$ has been manufactured with a zig-zag scan strategy: Onyx filaments were deposited in a zig-zag pattern rotated of $45^{\circ}$ to simulate the unidirectional pattern of a traditional laminate composite. This pattern also integrates a concentric contour fill of every single layer to give the panel an emphasized wall strength.

For the panel, 14 different layers have been used. Considering that each layer has a thickness of 150 microns, the resulting thickness of the entire panel is about $2 \mathrm{~mm}$. As observed in Fig.1, a scheme of the 3d-printed panel is proposed. It is shown that the chosen scan strategy affects the morphology of the surface of the substrate characterized. In fact, on the surface, the presence of gaps between the filaments oriented at $45^{\circ}$ can be noticed. 


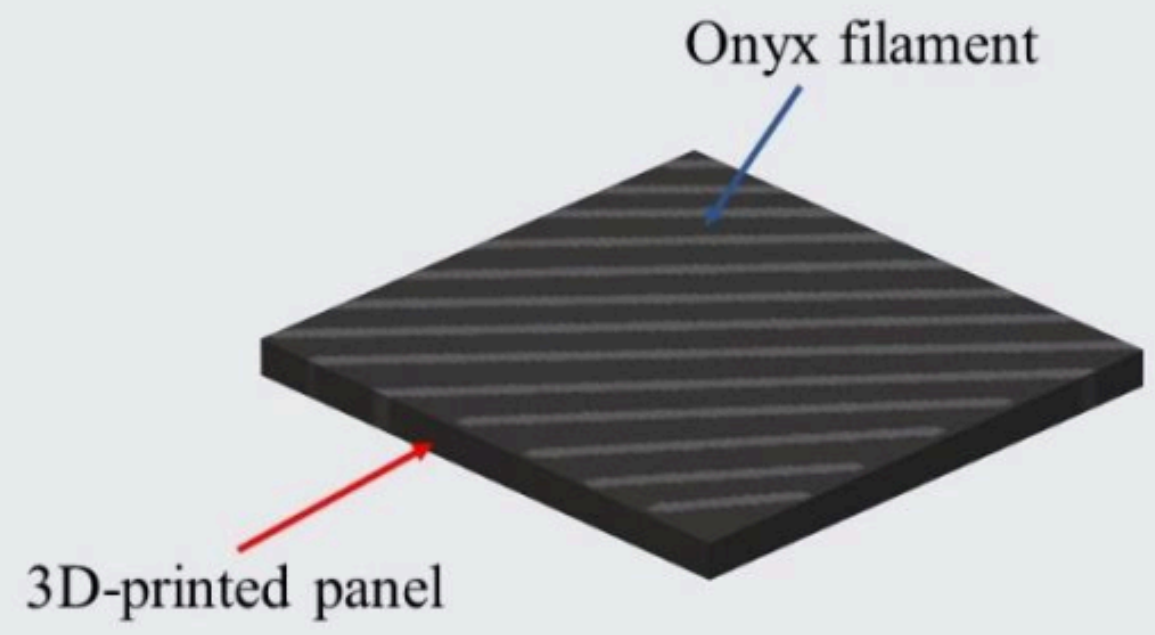

Fig.1 Scheme of the Onyx substrate

\subsection{Cold Spray Deposition Process}

Gas-atomized aluminum powder of a size dimension range from 15 to $45 \mu \mathrm{m}$ was chosen as feedstock material. It is widely accepted that the deposition of light materials such as aluminum is more achievable on composites and polymeric materials due to their minimal impact velocities[17] and this makes these types of powder more suitable for cold spray deposition. A Dymet 423 low-pressure cold spray (LPCS) system was used. As proposed by Małachowska et al. [18] LPCS equipment is successfully used for metal deposition on a thermoplastic substrate compared to the high-pressure one. Nitrogen was used as the carrier gas while the inlet gas pressure was maintained constant at 0.6 MPa. To analyze the influence of the process parameters on the deposition, two different inlet gas temperatures of $250^{\circ} \mathrm{C}$ and $350^{\circ} \mathrm{C}$ and two different standoff distances between the nozzle exit and the substrate of $10 \mathrm{~mm}$ and $20 \mathrm{~mm}$ were used. As summarized in Table 1., four different samples are provided with a combination of the above-mentioned parameters.

Table 1. Experimental Campaign

\begin{tabular}{cccc}
\hline Sample & Pressure $[\mathrm{bar}]$ & Temperature $\left[{ }^{\circ} \mathrm{C}\right]$ & SoD $[\mathrm{mm}]$ \\
\hline 1 & & 250 & 10 \\
2 & 6 & & 20 \\
3 & & 350 & 10 \\
4 & & & 20 \\
\hline
\end{tabular}




\subsection{Characterization of the Coating}

The coated panels were observed from both top and cross-sections direction. For cross-section observations, samples were cut perpendicularly to the sprayed surface by a precision hacksaw in small specimens of about $10 \mathrm{~mm}$ width and then mounted in a cold resin and polished with different grinding phases. Different observations, employing several types of equipment and preparations of the specimens, were carried out. Observations were made by using Hitachi TM 3000 SEM and Confocal Microscope Leica DCM3D Scan. To quantify the effectiveness of the deposition, as already done in other works $[19,20]$, the measure of the surface coverage, i.e. the quantity of the effective covered surface area has been proposed. Surface coverage measurements were made with ImageJ software through three different SEM acquisitions of the coating surface.

\section{Results and Discussion}

From a first visual inspection of the coating in Fig.2, it is evident that the deposition has been achieved for all the spray conditions and the coating is mostly homogenous with no evident damages, crack, or erosions of the polymer substrate. Besides, some interesting observations can be withdrawn. At the lower temperature of $250^{\circ} \mathrm{C}$, as shown in Fig.2 a) and Fig.2 b), some parts of the substrate have not been fully coated, some spots can be noticed, and few impact craters are present on the surface. On the other hand, at the higher temperature of $350^{\circ} \mathrm{C}$, as shown in Fig.2 c) and Fig.2 d), these empty spots are not observed, and all the parts of the surface appear to be covered. These observations suggest that when the temperature is higher the deposition efficiency is enhanced, and it could be assumed that the bonding between the powders and the substrate is increased. In fact, at the two distinct temperatures, the number of deposited particles was extremely different, then it was minor in the case of sprayed coatings at the lower temperature. The presence of uncovered areas and impact craters on the substrate may be caused by a low anchoring force between the sprayed powder and the substrate. As observed by Della Gatta et al [19] the presence of these impact craters on the polymer surface can be caused by the fact that the impact velocity of the aluminum particles does not reach the minimum critical velocity required for adhesion: particles impact onto the surface, deform or crack the surface and then rebound without stacking into the substrate. Anyhow, in all the cases, surface erosion has been negligible. 

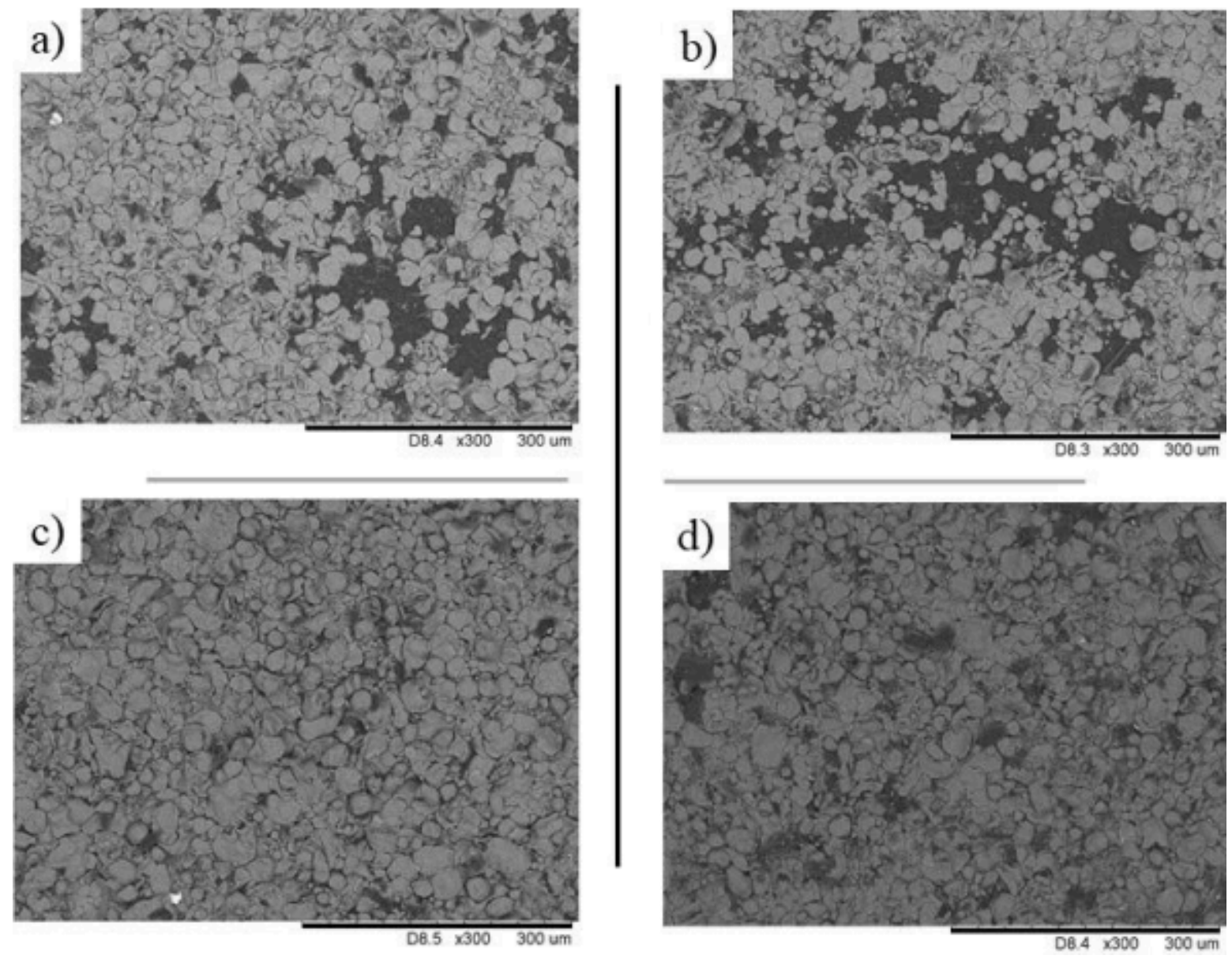

Fig. 2 Sem acquisition of deposits at a) $\mathrm{T}=250^{\circ} \mathrm{C}$ and $\left.\mathrm{So} D=10 \mathrm{~mm}, \mathrm{~b}\right) \mathrm{T}=250^{\circ} \mathrm{C}$ and $\left.\mathrm{SoD}=20 \mathrm{~mm}, \mathrm{c}\right) \mathrm{T}=350^{\circ} \mathrm{C}$ and $\mathrm{SoD}=10 \mathrm{~mm}$ and $\mathrm{d}) \mathrm{T}=350^{\circ} \mathrm{C}$ and $\mathrm{SoD}=20 \mathrm{~mm}$

For deeper analysis, in Fig.3 surface coverage results are shown and plotted against the inlet gas temperature for each SoD. For both the SoD it is evident an enhanced surface coverage, and thus a higher deposition efficiency when increasing the inlet gas temperature.

The highest value of surface coverage is reached at the temperature of $350^{\circ} \mathrm{C}$ at a fixed $\mathrm{SoD}$ of $10 \mathrm{~mm}$ while the minimum is reached at the temperature of $250^{\circ} \mathrm{C}$ at $\mathrm{SoD} 20 \mathrm{~mm}$. 


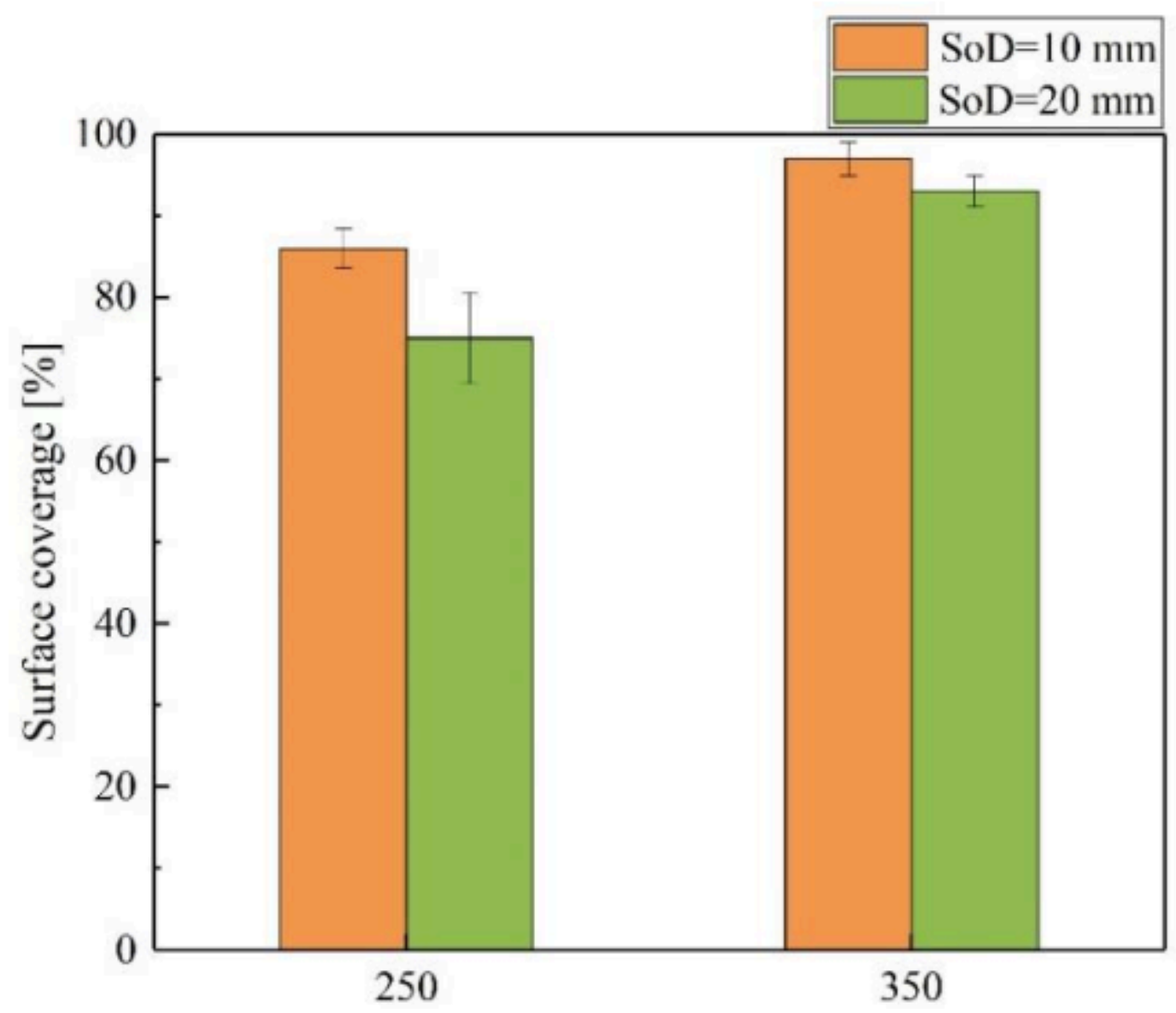

Fig.3 Surface coverage measurements.

From these observations, both process parameters of standoff distance and gas inlet temperature have a combined effect on the deposition. When the inlet gas temperature is higher the surface coverage is superior; at the same time, when the SoD is higher the surface coverage arises. Anyway, it is worth noticing that the lack of deposition is shown for both SoD at the lowest temperature, suggesting that the effect of the inlet gas temperature is more prominent than the effect of the standoff distance. In fact, as proved by Ganesan et al. [21], it is obvious that the increased process gas temperature results in higher particle velocity, in addition to the increased particle temperature and the consequent flattening of the particles, and deformation of the substrate is improved.

In the Materials and Method section, the manufacturing of the substrate has been shown and it has been highlighted that the superficial morphology reflects the stratification of the filaments. As resulted in other works [22], it is shown that the morphology of the substrate plays a key role in the particle deposition, leading to a higher mechanical anchoring of the particles into the substrate, and that makes the morphology of the 3d-printed surface more distinctive for the mechanical interlocking of the particles.

From the confocal analysis of the coatings shown in Fig.4, it is marked that the deposited coatings followed the pattern of the substrate. At a fixed inlet gas temperature of $250^{\circ} \mathrm{C}$, in Fig. 4 a) it is shown that at SoD of $10 \mathrm{~mm}$ the deposit follows the pattern of the substrate while at the SoD of $20 \mathrm{~mm}$, as shown in Fig4.b), the profile of the coating flattens, suggesting that particles deposit only in the cavities within the filaments. That means that at the SoD of $20 \mathrm{~mm}$ and the inlet gas temperature of $250^{\circ} \mathrm{C}$, particles have not enough energy and cannot adhere to each part of the substrate. In fact, in the cavities, as demonstrated by Kromer et al. [22], the bounce-off of the particles is limited, and also the 
particles with a velocity lower than the critical value can be mechanically interlocked to the substrate.

a)

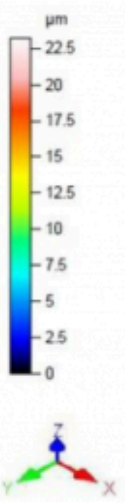

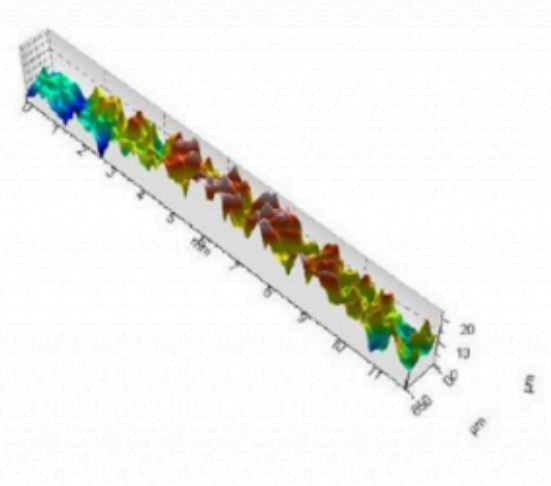

b)

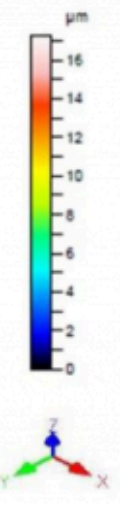

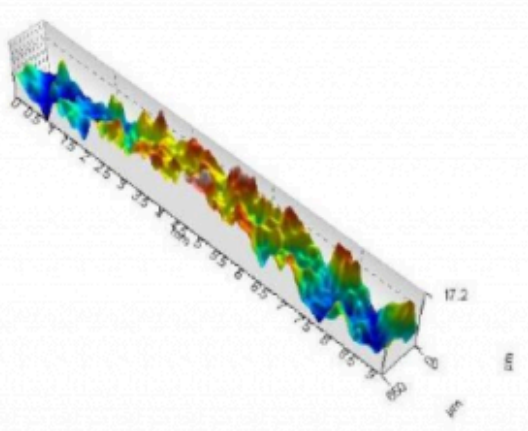

Fig 4. Confocal acquisitions of coatings at $250^{\circ} \mathrm{C}$ with a) $\mathrm{SoD}=10 \mathrm{~mm}$ and $\left.\mathrm{b}\right) \mathrm{SoD}=20 \mathrm{~mm}$.

a)

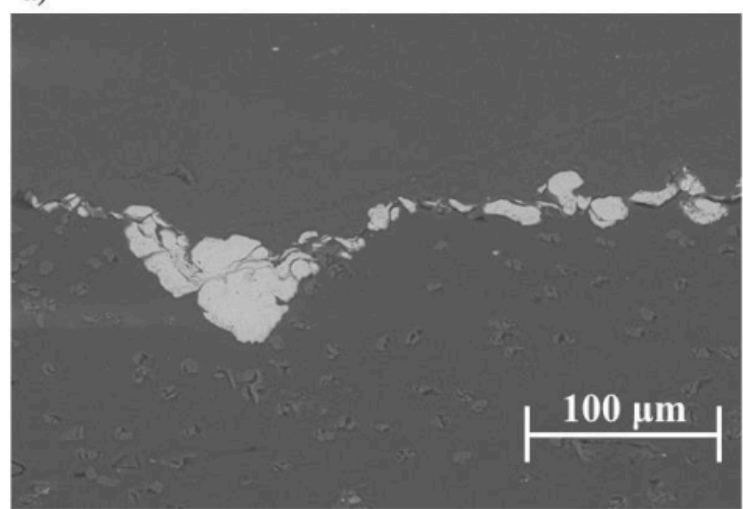

b)

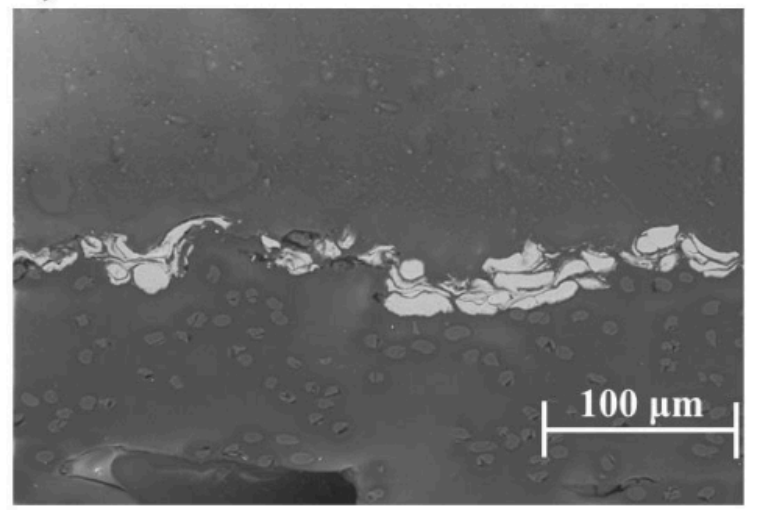

Fig 5. Sem images of coatings cross-sections at fixed $\mathrm{SoD}=10 \mathrm{~mm}$ and a) $\mathrm{T}=50^{\circ} \mathrm{C}$ and $\mathrm{b}$ ) $\mathrm{T}=350^{\circ} \mathrm{C}$

It is worth noticing that the deformation of the aluminum particles was dramatically improved with the increase in temperature, given the same inlet gas pressure and SoD distance. As observed by Steenkiste et al. [23] when gas temperature is higher, the particles have more energy and, after the impact, they deform more. At the same time, when the temperature is higher, the deformation of the thermoplastic polymer and the consequent embedment of the particles is enhanced. When the temperature is lower, the substrate has not heated enough to guarantee the deformation and the powder embedment [21].

\section{Conclusions}

Aluminum coatings were cold-sprayed on 3D-printed composites substrate by using low-pressure cold spray equipment 
at fixed inlet gas pressure and different inlet gas temperatures and standoff distances. The experimental outcomes reported and discussed in the previous sections can be summarized as follows:

- Al powders were successfully deposited on 3d-printed substrates under different processing conditions. The morphology of the substrate, characterized by the presence of repeated cavities, is found to be extremely useful to guarantee an enhanced mechanical anchoring of the particles with the substrates even at lower gas temperature or higher SoD distance.

- It was proved that when the standoff distance increases, the flattening ratio of the particles is reduced and consequently the coating height is higher.

- With cross-section observations, it has been highlighted that aluminum particles experienced more deformation when increasing the inlet gas temperature, suggesting an enhanced coating adhesion to the substrate. Otherwise, no thermal degradation of the polymer is observed.

\section{Bibliography}

[1] Mittal, KL. Metallized Plastics: Fundamentals and Applications. 1998.

[2] Preparation of Thin Metal Layers on Polymers. Preparation of Thin Metal Layers on Polymers, 2007, 47.

[3] Duguet, T. Senocq, F. Laffont, L. Vahlas, C. Metallization of polymer composites by metalorganic chemical vapor deposition of Cu: Surface functionalization driven films characteristics. Surface and Coatings Technology, 2013, 230, 254-9.

[4] Gritsenko, KP. Metal-polymer optical storage media produced by PECVD. Thin Solid Films, 1993, 227, 1-2.

[5] Rokni, MR. Feng, P. Widener, CA. Nutt, SR. Depositing Al-Based Metallic Coatings onto Polymer Substrates by Cold Spray. Journal of Thermal Spray Technology, 2019, 28, 1699-708.

[6] Viscusi, A. Astarita, A. Gatta, R Della. Rubino, F. A perspective review on the bonding mechanisms in cold gas dynamic spray. Surface Engineering, 2019, 35, 743-71.

[7] Archambault, G. Jodoin, B. Gaydos, S. Yandouzi, M. Metallization of carbon fiber reinforced polymer composite by cold spray and lay-up molding processes. Surface and Coatings Technology, 2016, 300, 78-86.

[8] Huang, G. Wang, H. Li, X. Xing, L. Zhou, J. Deposition efficiency of low pressure cold sprayed aluminum coating. Materials and Manufacturing Processes, 2018, 33, 1100-6.

[9] Che, H. Vo, P. Yue, S. Investigation of Cold Spray on Polymers by Single Particle Impact Experiments. Journal of Thermal Spray Technology, 2019, 28, 135-43.

[10] Ganesan, A. Yamada, M. Fukumoto, M. Cold spray coating deposition mechanism on the thermoplastic and thermosetting polymer substrates. J. Therm. Spray Technol., vol. 22, 2013, p. 1275-82.

[11] Gillet, V. Aubignat, E. Costil, S. Courant, B. Langlade, C. Casari, P. Knapp, W. Planche, MP. Development of low pressure cold sprayed copper coatings on carbon fiber reinforced polymer (CFRP). Surface and Coatings Technology, 2019, 364, 306-16.

[12] Viscusi, A. Astarita, A. Genna, S. Leone, C. On the influence of different superficial laser texturing on the deposition of powders through cold spray process. Transactions of the Institute of Metal Finishing, 2018, 96, 34-40. 
[13] Advani, SG. Hsiao, KT. Manufacturing techniques for polymer matrix composites (PMCs). 2012.

[14] Wang, X. Jiang, M. Zhou, Z. Gou, J. Hui, D. 3D printing of polymer matrix composites: A review and prospective. Composites Part B: Engineering, 2017, 110, 442-58.

[15] Sanei, SHR. Lash, Z. Servey, J. Gardone, F. Nikhare, CP. Mechanical properties of 3D printed fiber reinforced thermoplastic. ASME Int. Mech. Eng. Congr. Expo. Proc., vol. 12, 2019.

[16] Ghebretinsae, F. Mikkelsen, O. Akessa, AD. Strength analysis of 3D printed carbon fibre reinforced thermoplastic using experimental and numerical methods. IOP Conf. Ser. Mater. Sci. Eng., vol. 700, 2019.

[17] Che, H. Vo, P. Yue, S. Metallization of carbon fibre reinforced polymers by cold spray. Surface and Coatings Technology, 2017, 313, 236-47.

[18] Małachowska, A. Winnicki, M. Stachowicz, M. Korzeniowski, M. Metallisation of polycarbonates using a low pressure cold spray method. Surface Engineering, 2018, 34, 251-8.

[19] Della Gatta, R. Viscusi, A. Perna, AS. Caraviello, A. Astarita, A. Feasibility of steel powder deposition on composites through cold spray. Materials and Manufacturing Processes, 2021, 36, 281-91.

[20] Della Gatta, R. Viscusi, A. Perna, AS. Caraviello, A. Astarita, A. Cold spray process for the production of AlSi10Mg coatings on glass fibers reinforced polymers. Materials and Manufacturing Processes, 2021, 36, 106-21.

[21] Ganesan, A. Affi, J. Yamada, M. Fukumoto, M. Bonding behavior studies of cold sprayed copper coating on the PVC polymer substrate. Surface and Coatings Technology, 2012, 207, 262-9.

[22] Kromer, R. Danlos, Y. Aubignat, E. Verdy, C. Costil, S. Coating deposition and adhesion enhancements by laser surface texturing-metallic particles on different classes of substrates in cold spraying process. Materials and Manufacturing Processes, 2017, 32, 1642-52.

[23] Van Steenkiste, T. The role of particle temperature and velocity in cold spray coating formation. Cold Spray Mater. Depos. Process Fundam. Appl., 2007, p. 127-47.

PDF automatically generated on 2021-05-19 17:53:00

Article url: https://popups.uliege.be/esaform21/index.php?id=858

published by ULiège Library in Open Access under the terms and conditions of the CC-BY License

(https://creativecommons.org/licenses/by/4.0) 